\title{
Workplace gender discrimination and Consciousness of Sexual Violence in Female Workers - Centered on the mediating effect of consciousness of patriarchal gender roles
}

\author{
Junbi Kim, Okchai Choi ${ }^{1}$ \\ Department of Social Welfare, Jeonbuk National University
}

\begin{abstract}
Consciousness of Sexual Violence means how clearly one recognize that a sexual bullying is a violence, and Consciousness of Gender Roles means having different perceptions of the roles of males and females depending on gender. This study explored workplace gender discrimination experienced by female workers and observed the validation of the mediating effect of consciousness of patriarchal gender roles. The subjects were 2,370 female workers from sixth data(2016) of the Female Family Panel Survey conducted by the Korea Women's Development Institute. Structural Equation Modeling was adopted to examine measurement model and mediating effects of consciousness of patriarchal gender roles by using Bootstrapping method. The results showed that workplace gender discrimination experienced by female workers had a negative effect on their consciousness of sexual violence, and their consciousness of patriarchal gender roles had a negative effect on their consciousness of sexual violence. Also, their consciousness of gender roles partially mediated the relationship between workplace gender discrimination experienced by female workers and their consciousness of sexual violence. In other words, the more frequently female workers experience workplace gender discrimination, the higher their consciousness of patriarchal gender roles, and the higher their consciousness of patriarchal gender roles, the lower their consciousness of sexual violence. This suggests that individuals' consciousness of sexual violence is affected by their workplace and organization, and improving consciousness of gender roles can contribute to improving consciousness of sexual violence. The results suggested intervention measures for preventing sexual violence at the individual, workplace and organizational levels.
\end{abstract}

Keywords: Gender equality; Gender discrimination; Labor rights; Sexual values; Workplace culture

\footnotetext{
${ }^{1}$ Corresponding author: Professor in the Department of Social Welfare, Jeonbuk National Univer sity
} 